\title{
The Filter Imager SuFI and the Image Stabilization and Light Distribution System ISLiD of the Sunrise Balloon-Borne Observatory: Instrument Description
}

\author{
A. Gandorfer • B. Grauf • P. Barthol · T.L. Riethmüller • S.K. Solanki • B. Chares • \\ W. Deutsch · S. Ebert · A. Feller · D. Germerott · K. Heerlein · J. Heinrichs · \\ D. Hirche $\cdot$ J. Hirzberger $\cdot$ M. Kolleck $\cdot$ R. Meller $\cdot$ R. Müller $\cdot$ R. Schäfer • \\ G. Tomasch • M. Knölker • V. Martínez Pillet • J.A. Bonet • W. Schmidt • T. Berkefeld • \\ B. Feger $\cdot$ F. Heidecke $\cdot$ D. Soltau $\cdot$ A. Tischenberg $\cdot$ A. Fischer $\cdot$ A. Title $\cdot$ H. Anwand $\cdot$ \\ E. Schmidt
}

Received: 8 June 2010 / Accepted: 4 September 2010 / Published online: 3 November 2010

(C) The Author(s) 2010. This article is published with open access at Springerlink.com

\begin{abstract}
We describe the design of the Sunrise Filter Imager (SuFI) and the Image Stabilization and Light Distribution (ISLiD) unit onboard the Sunrise balloon borne solar observatory. This contribution provides the necessary information which is relevant to understand the instruments' working principles, the relevant technical data, and the necessary information about calibration issues directly related to the science data.
\end{abstract}

Keywords Instrumentation and data management

A. Gandorfer $(\varangle)$ · B. Grauf · P. Barthol · T.L. Riethmüller · S.K. Solanki · B. Chares · W. Deutsch •

S. Ebert · A. Feller · D. Germerott · K. Heerlein · J. Heinrichs · D. Hirche · J. Hirzberger · M. Kolleck ·

R. Meller $\cdot$ R. Müller $\cdot$ R. Schäfer $\cdot$ G. Tomasch

Max-Planck-Institut für Sonnensystemforschung, Max-Planck-Straße 2, 37191 Katlenburg-Lindau,

Germany

e-mail: gandorfer@mps.mpg.de

M. Knölker

High Altitude Observatory, P.O. Box 3000, Boulder, CO 80301, USA

V. Martínez Pillet · J.A. Bonet

Instituto de Astrofísica de Canarias, C/ Via Láctea, s/n, 38205 La Laguna, Tenerife, Spain

W. Schmidt · T. Berkefeld · B. Feger · F. Heidecke - D. Soltau · A. Tischenberg · A. Fischer Kiepenheuer-Institut für Sonnenphysik, Schöneckstraße 6, 79104 Freiburg, Germany

A. Title

Lockheed Martin Solar and Astrophysics Laboratory, Bldg. 252, 3251 Hanover Street, Palo Alto, CA 94304, USA

H. Anwand

Institut für Astrophysik, Georg-August-Universität, Friedrich-Hund-Platz 1, 37077 Göttingen, Germany

E. Schmidt

Ingenieurbüro für Optikentwicklung, Amalienstraße 12, 85737 Ismaning, Germany 


\section{Introduction}

Sunrise is a balloon-borne solar observatory with a telescope with an aperture of $1 \mathrm{~m}$, working in the visible and near ultraviolet spectral domain. The main scientific goal of Sunrise is to understand the structure and dynamics of the magnetic field in the atmosphere of the Sun.

The Sunrise focal-plane science instrumentation consists of a Fabry-Pérot filter magnetograph, and a phase-diversity assisted filter imager working in the near UV, which takes particular advantage of the observing conditions in the stratosphere.

Sunrise provides high resolution ultraviolet images of the photosphere and chromosphere, as well as Doppler- and magnetograms with an unprecedented spatial resolution, down to 0.15 arcsec on the solar surface.

Sunrise is a joint project of the Max-Planck-Institut für Sonnensystemforschung (MPS), Katlenburg-Lindau, with the Kiepenheuer-Institut für Sonnenphysik (KIS), Freiburg, Germany, the High-Altitude Observatory (HAO), Boulder, USA, the Lockheed-Martin Solar and Astrophysics Lab. (LMSAL), Palo Alto, USA, and the Spanish IMaX consortium consisting of the Instituto de Astrofísica de Canarias (IAC), the Instituto de Astrofísica de Andalucia (IAA), the Instituto Nacional de Técnica Aeroespacial (INTA), and the Grupo de Astronomía y Ciencias del Espacio (GACE).

The first stratospheric long-duration balloon flight of Sunrise took place in Summer 2009 from the Swedish Esrange station. For an overview on the Sunrise project, instruments, and mission we refer to Barthol et al. (2010). In the present paper we describe one of the two science instruments, the Sunrise Filter Imager (SuFI) and the Image Stabilization and Light Distribution (ISLiD) system, which were designed as a single, integral unit.

\subsection{Science Requirements and Design Drivers}

The science goals of Sunrise are described in Barthol et al. (2010) and are not repeated here. Driven by these science goals, the Sunrise instruments are required to provide images of the magnetic structure and measurements of the magnetic field, the flow velocity, and thermodynamic properties of the plasma

- with a spatial resolution of the order of 0.1 arcsec

- on a field of view of $50 \times 50$ arcsec (in the magnetograms)

- over a sufficiently long time to follow the evolution of magnetically active regions (i.e., several days), and

- simultaneously in different heights of the solar atmosphere.

This led to the concept of a telescope of $1 \mathrm{~m}$ aperture operating in the visible and UV spectral ranges (down to $\simeq 210 \mathrm{~nm}$ ), with in-flight alignment capability, equipped with a filter imager and an imaging magnetograph, on a long-duration stratospheric balloon flight in the framework of NASA's LDB program.

\subsection{Sunrise Post-Focus Instrumentation}

The Sunrise post-focus instrumentation consists of four units, two of which are science instruments, the other two are system units for image stabilization and light distribution.

i) ISLiD

The Sunrise science requirements demand simultaneous observations of the post-focus instruments. This is provided by ISLiD, the Image Stabilization and Light Distribution system of Sunrise, which will be described in detail in Section 2. ISLiD contains a fast 
tip-tilt mirror, which is controlled by a correlating wavefront sensor (CWS, Berkefeld et al., 2010). ISLiD is based on dichroic beam-splitters, which guide the different wavelength bands to the individual instruments in the most efficient way. Part of the light, which is not used for scientific analysis, is fed to the CWS. In this way, simultaneous observations are possible with maximum photon flux in each channel.

ii) $\mathrm{SuFI}$

The Sunrise Filter Imager (SuFI) samples the photosphere and chromosphere in distinct wavelength bands. The channel at $214 \mathrm{~nm}$ allows studies of the upper photosphere and lower chromosphere at a theoretical angular resolution of 0.05 arcsec (corresponding to $35 \mathrm{~km}$ on the Sun). Solar radiation in this wavelength range is important for the stratospheric ozone household. The $\mathrm{OH}$-band at $313 \mathrm{~nm}$ and the $\mathrm{CN}$-band at $388 \mathrm{~nm}$ provide high intensity contrast, and thus sensitivity to the thermal structure of the photosphere and its embedded magnetic field structure. The Ca II $\mathrm{H}$ line (singly ionized calcium) at $397.6 \mathrm{~nm}$ is an excellent thermometer for the chromospheric temperature structure.

iii) IMaX

The Imaging Magnetograph eXperiment for Sunrise (IMaX, Martínez Pillet et al., 2010) is an imaging vector magnetograph based upon a tunable narrow-band Fabry - Pérot filter. The instrument provides fast-cadence two-dimensional maps of the magnetic vector, the line-of-sight velocity, and continuum frames with high spatial resolution. IMaX takes polarized images in two to five narrow wavelength bands in either wing of the photospheric spectral line of Fe I (neutral iron) at $525.02 \mathrm{~nm}$.

iv) CWS

The Correlation tracker and Wavefront Sensor (CWS, Berkefeld et al., 2010) is used for two purposes, namely for precision image stabilization and guiding, and to control proper alignment of the telescope.

\section{Design of the SuFI/ISLiD Instrument}

\subsection{Optical Design Drivers and Design Philosophy}

ISLiD is a complex optical instrument that has to simultaneously fulfill very different tasks. Firstly, it has to stabilize the incoming beam from the telescope to provide stable output images for the science instruments and the CWS. Secondly, it must divide the incoming light into the wavelength bands required by the instruments, and thirdly, it must feed the instruments with the corresponding wavelengths by providing optical interfaces at given positions and with given directions, plate scale, and pupil position. Since Sunrise is a high resolution observatory, two requirements must be satisfied: Firstly, the optical system must allow for diffraction-limited performance under all environmental conditions that might arise during flight. Secondly, the image stabilization system must damp the residual pointing error (RPE) to a value which does not degrade the high intrinsic image sharpness by image smear.

It is evident that the different instruments that are fed by ISLiD have individual requirements, which makes it necessary to sort the instruments in a priority list - not in the sense of ranking their scientific importance for the mission, but rather from the point of view of their intrinsic challenges which they impose on the optical design of ISLiD.

Figure 1 shows schematically the Sunrise optical system and lists the key optical requirements of the individual units from the ISLiD point of view. The most severe requirement of each category is highlighted: the largest simultaneous field of view, the broadest wavelength 


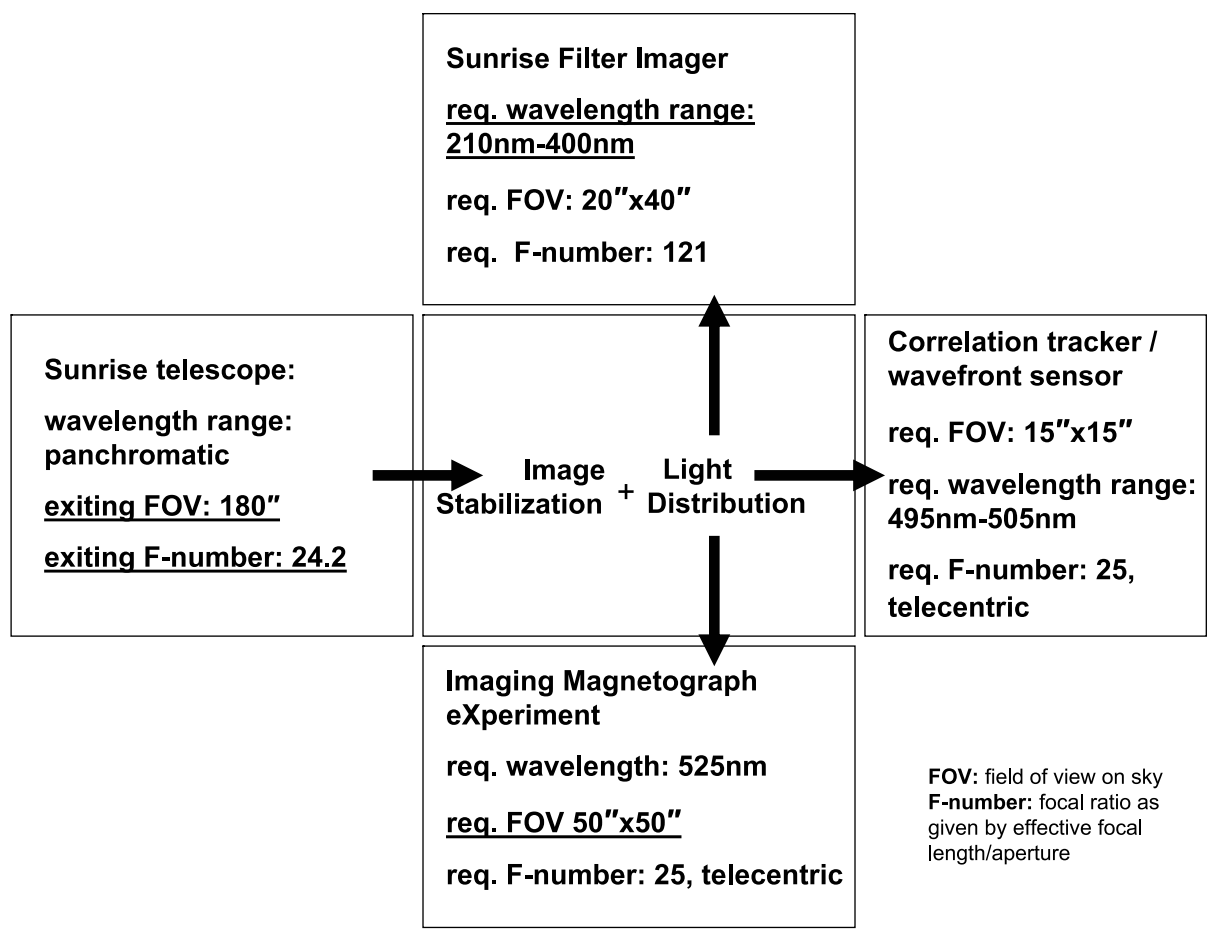

Figure 1 Scheme of the Sunrise optical system with the relevant optical key requirements from the point of view of ISLiD. Requirements driving the ISLiD design are underlined. See text for details.

band, the shortest absolute wavelength, and the fastest working aperture given as effective F-number. Not listed here are any further requirements such as beam direction, image position, or pupil position. Since all instruments were designed to operate at their individual diffraction limit, which is set by the telescope aperture and the specific working wavelength of the instrument, the most critical instrument path is imposed by the SuFI instrument, which aims at a diffraction limit at $214 \mathrm{~nm}$. Also the wavelength bandwidth of SuFI is by far the broadest, spanning almost a factor of two. The SuFI science focus needs a five times larger plate scale than the telescope exit focus (in order to allow for three-pixel oversampling of the point spread function at $214 \mathrm{~nm}$ ), and therefore a magnification of five is needed from telescope F2 to SuFI science focus. It is evident that diffraction-limited performance at these short wavelengths can only be achieved if the number of optical surfaces is kept to a minimum.

To this end SuFI is completely incorporated into ISLiD, making the SuFI image path an intrinsic part for all instruments. All instrument feeds make use of the SuFI path, which is designed to be diffraction limited at $214 \mathrm{~nm}$, and is therefore uncritical for the longer wavelengths. The separation of the UV light is done after the beam stabilization, and close to SuFI focus, mitigating the disturbing influence of the beam-splitter plates, which must be expected to be bent out of shape by their complex coatings. After the separation of the UV light, each of the remaining instruments is fed by a dedicated optical feed providing the required magnification, image, and pupil position. Since CWS and IMaX are operating in the visible range and can be considered as monochromatic, this can best be achieved by dedicated lens optics. 
In order to achieve diffraction-limited performance of the SuFI path, the following strategy was employed: First, the number of surfaces needed to reimage (magnify) telescope F2 onto SuFI science focus must be minimized. Further, the working field of view (FOV) of $\mathrm{SuFI} / \mathrm{ISLiD}$ must be determined by the largest instrument field $\left(50^{\prime \prime} \times 50^{\prime \prime}\right)$ only and not by the very large telescope exit field of $180 \mathrm{arcsec}$, which is only required for image motion compensation.

This can be achieved by stabilizing the image before the light enters the reimaging optics: A field lens near the telescope focus images the telescope aperture onto a plane folding mirror, which is actuated by a fast piezo system and acts as fast tip-tilt mirror. Behind this mirror, we only have to consider the reduced FOV of 50 arcsec in the design of the imaging optics. With that, the remaining key requirements for the reimager are: A FOV of 50 arcsec, a working F-number of 24.2 in the incoming path, and an effective F-number of 121 in the exit path. This allows for a design with two spherical mirrors only, which is optimum in terms of design stability. The spherical mirrors are well polishable and not very sensitive to alignment errors, a fact that is of particular importance for a balloon mission, where both, gravity load and thermoelastic deformations change with telescope elevation.

\subsection{Optical Design}

We have chosen the design of a modified Schwarzschild microscope. The principle is sketched in Figure 2(a). Schwarzschild systems are common in ultraviolet microscopy. They can have large numerical apertures and cover quite a large FOV. For a slow optical system with an F-number of 24.2 only, the used aperture can be decentered and an off-axis configuration can be used, thus eliminating any obscuration while preserving the superb optical performance.

The design steps from the principle of a Schwarzschild microscope to the final SuFI arrangement are depicted in Figure 2. From the original Schwarzschild configuration (Figure 2(a)) only a small part of the mirror is used, corresponding to an entrance F-number of 24.2. This part of the mirror is decentered (Figure 2(b)). In addition the system is used off-axis (object and image are not on the optical axis given by the connection line of the mirror centers). As Figure 2(c) shows, the incoming beam is folded by a small plane mirror, which is used as image motion compensator (tip-tilt mirror). It must therefore sit in a pupil of the system. This is achieved by placing a field lens near the telescope secondary focus. In Figure 2(d) we show that a dichroic beam-splitter plate separates the wavelengths below $450 \mathrm{~nm}$ by reflection, while transmitting the longer wavelengths. A set of interference filters in a filter wheel selects the working wavelength of SuFI. Another folding mirror sends the light to the CCD. In front of the CCD the phase-diversity image doubler (see Section 2.3) is placed.

All components except the two spherical mirrors and the tip-tilt mirror are close to focus and therefore optically not very critical. This is of key importance especially for non-perfect optical surfaces such as the dichroic beam-splitter and the interference filters (which are usually bent by the coating), as well as the 45 degree folding mirror. For these elements, the surface quality requirements can be somewhat relaxed without compromising the overall performance of the system.

\subsection{Phase-Diversity Capability}

Phase diversity is a technique for wavefront measuring and subsequent image restoration (Paxman, Schulz, and Fienup, 1992; Löfdahl and Scharmer, 1994). Two images of the same 


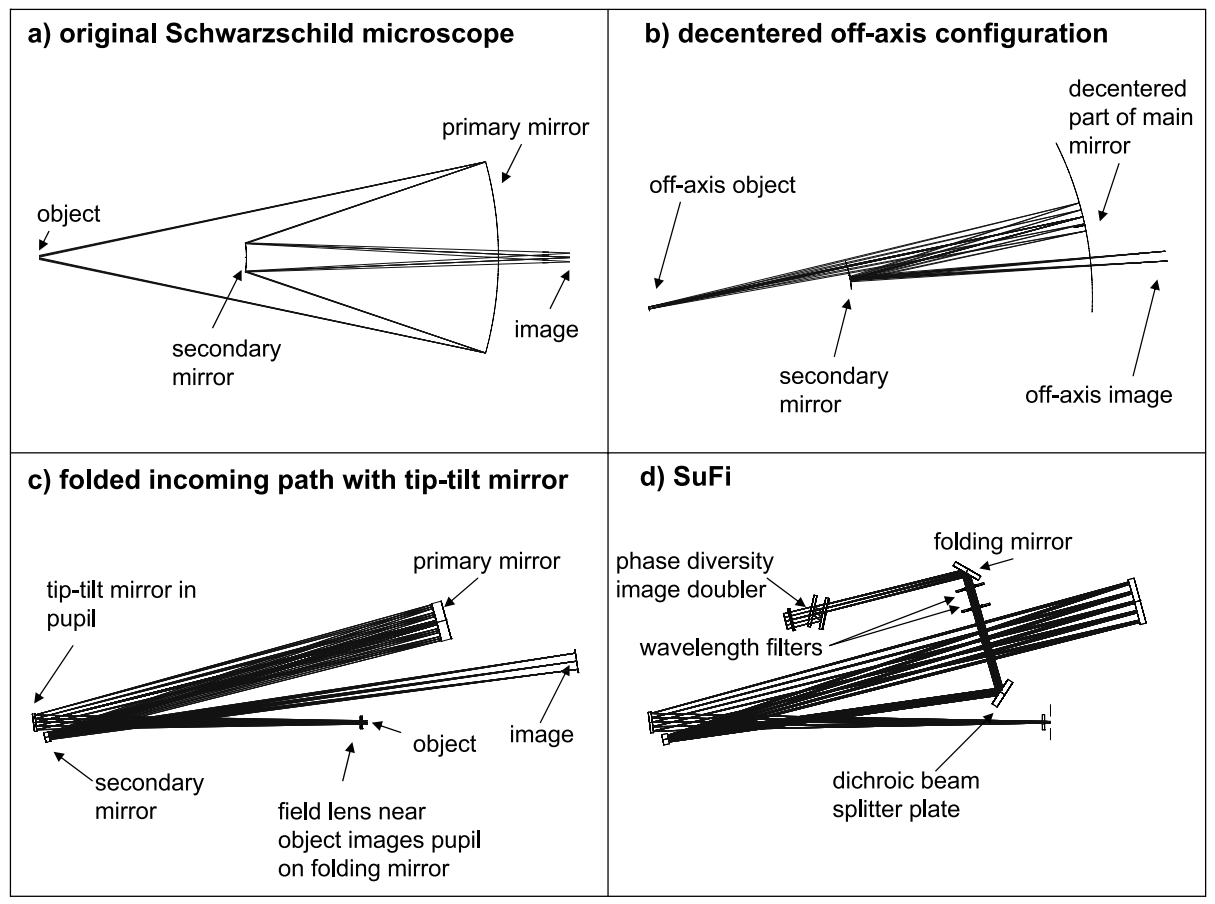

Figure 2 Optical design of SuFI: (a) Principle of a Schwarzschild microscope. The magnification of the sketched example design is 5, as in the SuFI instrument. (b) Sketch of the off-axis decentered pupil configuration. The system is stopped down to an entrance F-number of 24.2, yielding an exiting F/121 beam. (c) Folded configuration of the Schwarzschild system. A Lithosil field lens near the object plane images the telescope pupil on the folding mirror, which can now be used as a fast tip-tilt mirror. It compensates image motion in the object plane providing a fixed scene in image plane. (d) Final optical design of SuFI. The exiting beam of the Schwarzschild microscope is folded by a dichroic beam-splitter plate, which reflects all wavelengths below $450 \mathrm{~nm}$. The folded beam passes a double filter set for wavelength selection before the beam is folded again by a UV enhanced aluminum mirror. The phase-diversity image doubler is placed in front of the science focal plane. It provides a focussed and an out-of-focus image of the object side-by-side on the CCD detector.

object, which are taken simultaneously at two distinct focal positions (usually one image in best focus, the second defocussed such as to create an additional wavefront curvature of typically one wave) allow the wavefront in the exit pupil to be sampled at two distinct focal positions. From these two measurements, the object and the aberrations of the system can be retrieved. While for static objects and aberrations the two images can be taken sequentially, for a dynamic object like the Sun the two images must be taken simultaneously. Phasediversity capability was incorporated in Sunrise to correct for residual aberrations due to thermoelastic deformations of the telescope during flight. To this end, the light path includes a phase-diversity image doubler (see Figure 3). It resides directly in front of the focal plane and consists of two plane-parallel Suprasil plates, which are coated with dedicated highreflectivity and anti-reflective coatings on both sides. The second plate is coated on one half of its area with a 50/50 beam-splitter coating, that transmits half of the incoming radiation, while reflecting the other half. This leads to two images lying side-by-side on the focal plane: one image that has passed both glass plates without any reflection, and another image, which has undergone two additional reflections, the first at the beam-splitter plate, the second at 


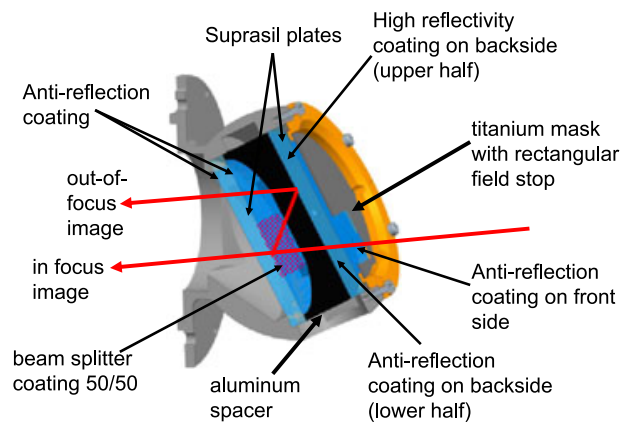

Figure 3 Design of the phase-diversity image doubler. A field stop in front of the two glass plates selects the useable field. Half of the light is transmitted through both plates directly and forms the in-focus image, while the other half is optically delayed by two reflections at the specially coated inner surfaces. The path difference is $28.15 \mathrm{~mm}$, corresponding to an additional wavefront curvature of approx. half a wave at $388 \mathrm{~nm}$ and approx. one wave at $214 \mathrm{~nm}$. The two images are laterally separated by $12.5 \mathrm{~mm}$, half the size of the CCD.

the backside of the first plate. The distance and the angle of the plates are chosen such that the path difference between the images is $28.15 \mathrm{~mm}$, while the lateral separation is $12.5 \mathrm{~mm}$, corresponding to half the CCD size. For the F/121 beam this path difference creates a defocus of approximately one wave at $214 \mathrm{~nm}$ and half a wave at $388 \mathrm{~nm}$.

\subsection{IMaX and CWS Feed Path}

Since the instruments have no internal focusing capabilities, ISLiD must ensure that the exit foci for SuFI, CWS, and IMaX are all kept well in focus under all environmental circumstances. Focusing of the entire post-focus assembly is done by focusing the telescope in F2 by moving the telescope secondary mirror (Barthol et al., 2010). The error signal for the focus position is provided by the CWS instrument (Berkefeld et al., 2010). It is therefore indispensable that the CWS and IMaX feed paths are identically affected by thermoelastic distortions of the post-focus instrument platform and by thermo-optic effects within ISLiD. This is achieved by bringing the CWS optical interface into close vicinity of the IMaX interface, and by using identical optical components for both paths, which are separated by a beam-splitter plate. The coating of this plate reflects a band centered at $525 \mathrm{~nm}$ to IMaX while transmitting a band centered around $500 \mathrm{~nm}$ to CWS.

Both paths start from a common achromatic field lens assembly in the focus of the Schwarzschild microscope, which has been corrected for astigmatism and coma induced by the SuFI beam-splitter plate by inserting a wedged corrector plate (see Figure 4). The field lens forms a real image of the instrument pupil. A second lens assembly reimages the Schwarzschild focus with the required plate scale (demagnification by a factor of 4.85), while creating a pupil image at infinity, as required by the instruments (telecentric exit paths). The full ISLiD optical design showing the ISLID-IMaX-CWS feed paths is depicted in Figure 4. Figure 5 shows the mechanical implementation of SuFI/ISLiD looking onto the SuFI compartment, while Figure 6 depicts the view from the other side, allowing a view onto the CWS and IMaX feed path implementation.

Besides the aspects discussed so far, the IMaX feed path must conserve the high polarimetric efficiency of the IMaX instrument. This implies that ISLiD must not produce significant linear polarization. Since in the design of ISLiD the possibility to feed an additional 


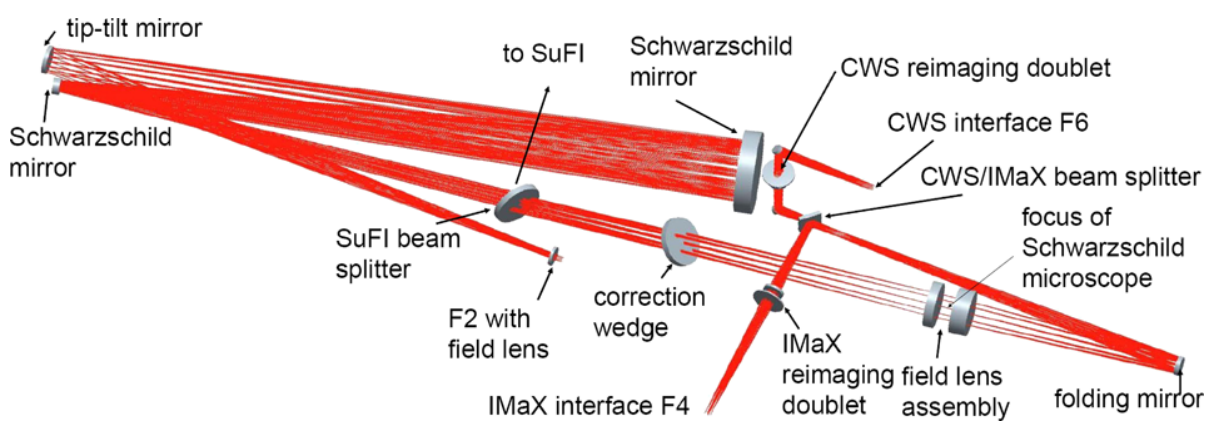

Figure 4 Optical design of the ISLiD-IMaX and ISLiD-CWS feed paths. The exiting beam of the Schwarzschild microscope is transmitted by a dichroic beam-splitter plate, which reflects all wavelengths below $450 \mathrm{~nm}$ to SuFI. A wedged corrector plate is used to compensate for astigmatism and coma induced by the SuFI beam-splitter. In the Schwarzschild focus a field lens assembly is placed, creating a real pupil image. In the folded path a second lens doublet reimages the Schwarzschild focus at the required position, while sending the pupil to infinity to fulfill the requirement of telecentricity.

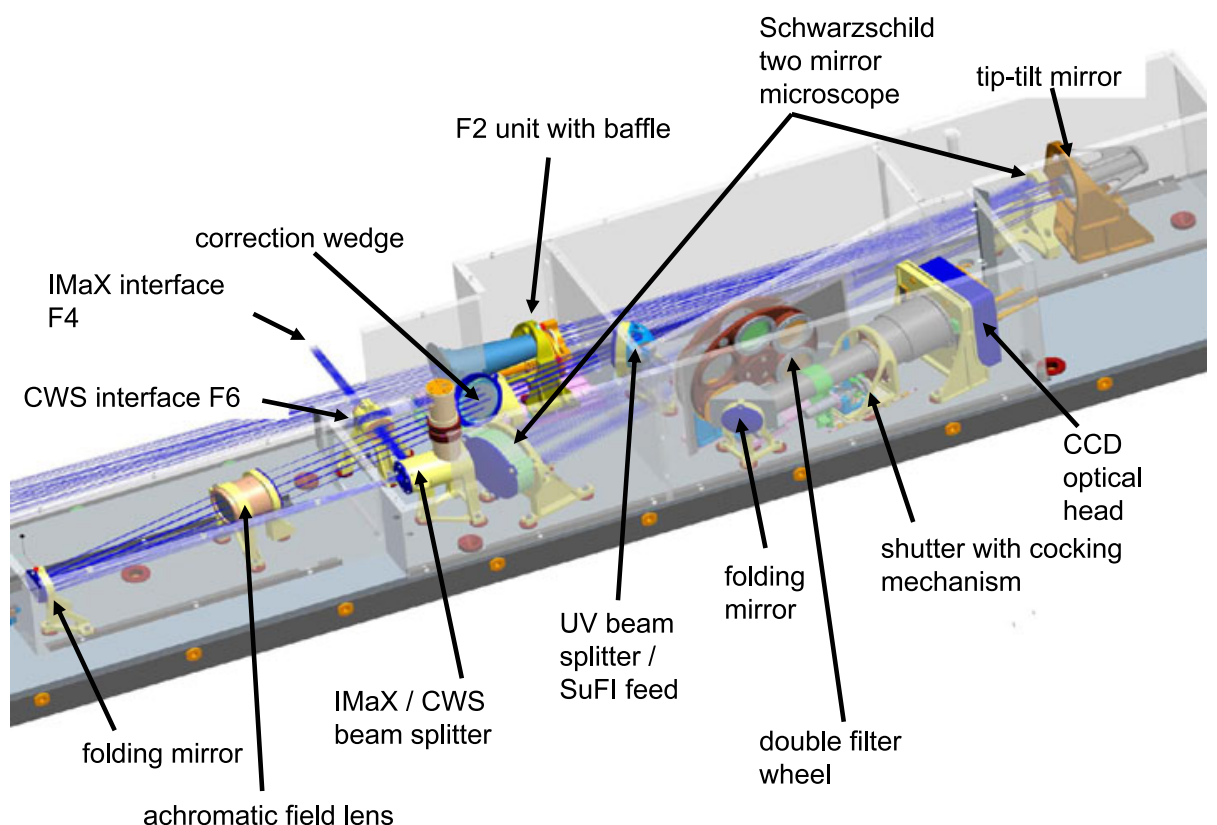

Figure 5 CAD overview of the ISLiD/SuFI optics unit, showing the SuFI path. Housing is shaded to provide insight into the compartments.

instrument in future is foreseen (a near IR full Stokes spectropolarimeter with a working wavelength at $854.2 \mathrm{~nm}$ ), ISLiD was designed for minimum linear polarization at $525 \mathrm{~nm}$ and $854 \mathrm{~nm}$. This was achieved by using metallic surfaces close to normal incidence and by a careful design of the beam-splitter coatings. 


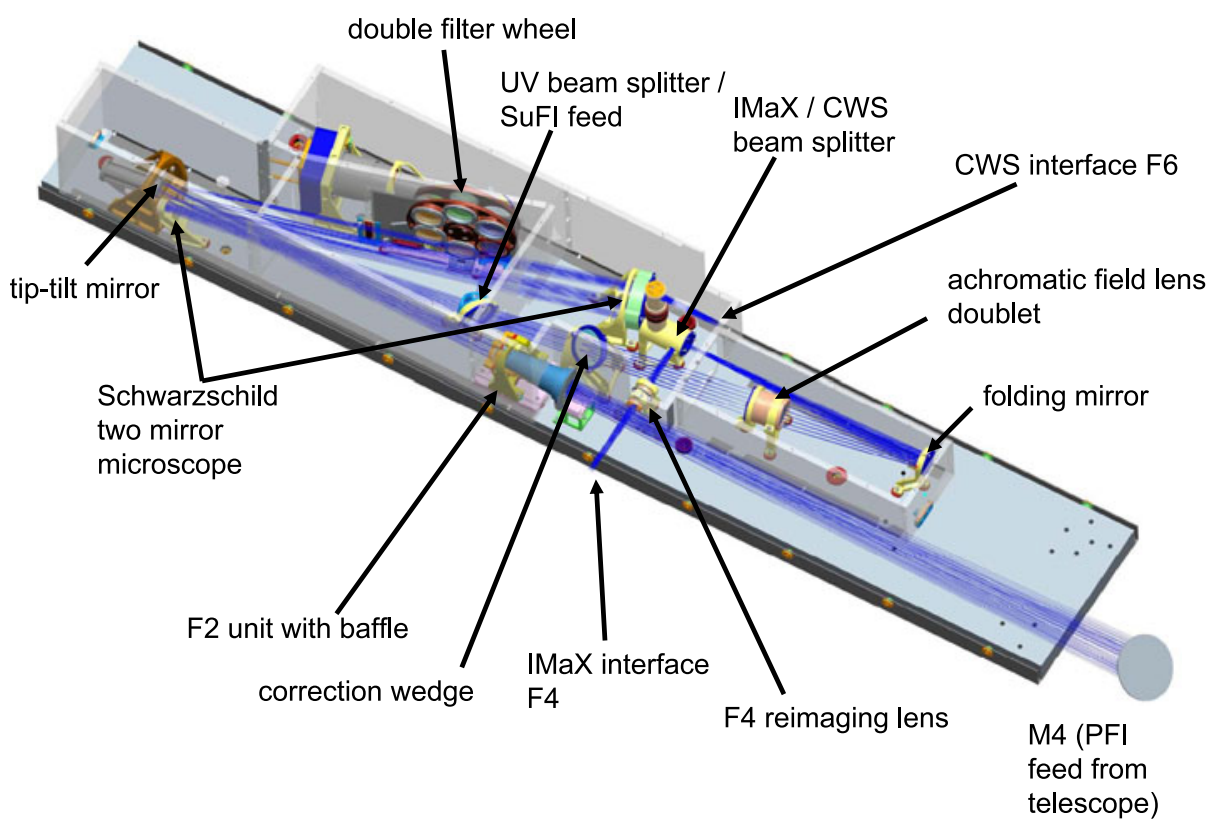

Figure $6 \mathrm{CAD}$ overview of the ISLiD/SuFI optics unit. Light from the telescope is fed by folding mirror M4 into the system. Telescope secondary focus is at F2, where the field is stopped down to 180 arcsec. The optical path of ISLiD is seen with the foci F4 and F6 feeding IMaX and CWS, respectively.

\subsection{ISLiD Mechanisms: F2 Unit and Tip-Tilt Stage}

ISLiD contains two mechanisms related to the CWS unit: The tip-tilt mirror stage and a calibration filter wheel in F2. The tip-tilt stage is a fast piezo-stage (Physik Instrumente PI $\mathrm{S} 330 \mathrm{~K} 032)$ with a range of \pm 50 arcsec on the sky. It is described in detail in Berkefeld et al. (2010).

The F2 calibration unit (see Figure 7) is a motorized filter wheel with three positions: An open position, a closed position for dark exposures, and a pinhole, which is used to monitor the relative alignment of the science instrument fields, and which is needed for CWS internal calibration during flight.

\subsection{SuFI Mechanisms: Filter Wheel and Shutter}

\subsubsection{Wavelength Selection}

The spectral bands to be observed by SuFI are summarized in Table 1 .

The sunlight intensity decreases strongly toward shorter wavelengths so that in the case of the UV wavelengths the suppression of unwanted contributions from leakage at longer wavelengths is only achievable by combining two filters at each filter position. A single specially blocked filter is sufficient only for the $\mathrm{Ca}$ II $\mathrm{H}$ line.

The filters are combinations of dielectric interference pass bands with color glass blocking, custom made by Barr Associates. All dielectric coatings have been applied using ionassisted deposition (IAD) techniques, which greatly helps in reducing the temperature sensitivity of the bandpass down to $0.005 \mathrm{~nm} \mathrm{~K}^{-1}$. This is of particular importance in a balloon mission, for which quite a large temperature range must be assumed during flight. 
Figure 7 CAD design of the F2 calibration unit. The field lens is mounted onto the same mount.
Table 1 Filters and typical exposure times used in the SuFI instrument.

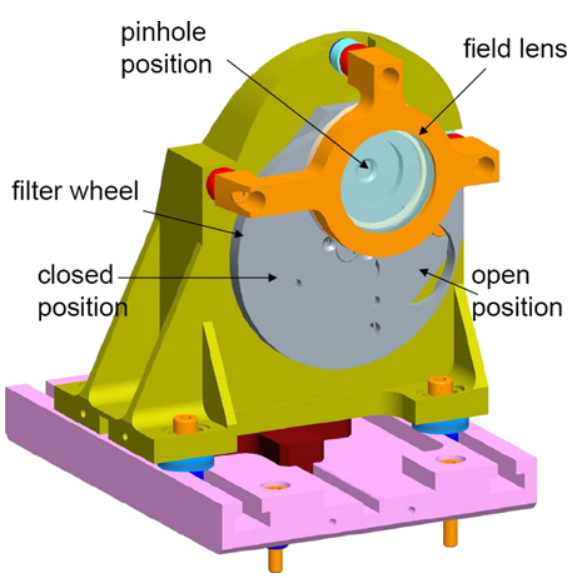

\begin{tabular}{|c|c|c|}
\hline \multirow[t]{4}{*}{$214 \mathrm{~nm}$ continuum } & center wavelength & $214 \mathrm{~nm}$ \\
\hline & FWHM & $10 \mathrm{~nm}$ \\
\hline & number of filters & 2 \\
\hline & exp. time & $30 \mathrm{~s}$ \\
\hline \multirow[t]{4}{*}{$300 \mathrm{~nm}$ continuum } & center wavelength & $300 \mathrm{~nm}$ \\
\hline & FWHM & $5 \mathrm{~nm}$ \\
\hline & number of filters & 2 \\
\hline & exp. time & $300 \mathrm{~ms}$ \\
\hline \multirow{4}{*}{$\begin{array}{l}312 \mathrm{~nm} \text { continuum } \\
\text { with lines of } \mathrm{OH}\end{array}$} & center wavelength & $312 \mathrm{~nm}$ \\
\hline & FWHM & $1.2 \mathrm{~nm}$ \\
\hline & number of filters & 2 \\
\hline & exp. time & $200 \mathrm{~ms}$ \\
\hline \multirow[t]{4}{*}{$388 \mathrm{~nm} \mathrm{CN}-$ band head } & center wavelength & $388 \mathrm{~nm}$ \\
\hline & FWHM & $0.8 \mathrm{~nm}$ \\
\hline & number of filters & 2 \\
\hline & exp. time & $100 \mathrm{~ms}$ \\
\hline \multirow[t]{4}{*}{397 nm Ca II H } & center wavelength & $396.8 \mathrm{~nm}$ \\
\hline & FWHM & $0.18 \mathrm{~nm}$ \\
\hline & number of filters & 1 \\
\hline & exp. time & $1 \mathrm{~s}$ \\
\hline
\end{tabular}

The tiny optical thickness variations of the different filter sets are well within the depth of focus of the F/121 beam.

The filters are placed approx. $25 \mathrm{~cm}$ in front of the focal plane, which for the $\mathrm{F} / 121$ beam is sufficient to prevent pinholes and other effects of the filters to be imaged onto the $\mathrm{CCD}$, while at the same time being close enough to make the image insensitive to wavefront deformations induced by the filters.

In order to minimize the induction of any torque into the instrument the two filters are mounted in two parallel separate filter wheels, which are driven by a common drive system in opposite directions. Each of the filter wheels has six positions. The filters are mounted 
with an inclination of 0.5 degrees to avoid ghost images. Between the two filter wheels, a baffle is placed, which is part of the inner baffling/housing system (see Section 2.8) that optically seals the optical path after wavelength selection.

The six positions of the filter wheels are used as follows: The four filter pairs $(214 \mathrm{~nm}$, $300 \mathrm{~nm}, 312 \mathrm{~nm}$, and $388 \mathrm{~nm}$ ) are arranged in such a way that the corresponding filters of the two wheels are always in the light beam at the same time. The single Ca II H filter $(397 \mathrm{~nm})$ is placed in the "sunny side" filter wheel (for stray light reasons) whereas the corresponding camera side filter wheel position is left empty. The remaining filter position contains a combination of lenses and neutral density filters, which image the tip-tilt mirror and thus the pupil onto the CCD. This feature was mainly needed during the PFI integration on top of the telescope, but provided also a monitor of the pupil (which is sensitive to the relative alignment of ISLiD with respect to the telescope) during flight.

Sunrise science requires series of alternating images of the solar photosphere and chromosphere with a high cadence. This means that changes between 397 and $300 \mathrm{~nm}$, between 397 and $388 \mathrm{~nm}$, and between 214 and $313 \mathrm{~nm}$ must be done as fast as possible. This can only be achieved if the respective filter combinations are placed at neighboring filter wheel positions.

The filter wheel is operated such that after one wavelength cycle the whole wheel is brought back to the first filter position not by continuation in the same rotation direction (+60 degrees), but by backward rotation ( -300 degrees). This ensures a higher positioning accuracy even after many thousand cycles as expected during a several day mission.

\subsubsection{Mechanical Shutter and Exposure Control}

Since the full area of the SuFI CCD is used for exposure, and thus the CCD cannot be operated in frame transfer mode, a separate mechanical shutter is used for exposure control. This shutter is placed near the focal plane in front of the phase-diversity image doubler. After different commercially available shutters had been tested, a Nikon dual-blade focalplane shutter as used in the Nikon D2H type cameras was selected (see Figure 8). It allows for fast exposures, exposes the covered area evenly and is easy to control. However it needs a mechanism for cocking.

The cock mechanism is shown in Figure 9. A motor with an attached 76:1 gear drives a spiral, operating a rocker. The rocker has a wheel where it is operated by the spiral. On the other end it operates the cocking lever of the Nikon shutter. There is a small Vespel part between the rocker and the shutter cock lever to reduce the point pressure and wear of the metal surfaces.

In order to mitigate disturbing effects by shutter cocking and release on the instrument, the whole shutter assembly is mounted on spring-loaded steel wires, which significantly damps the mechanical shock load on the ISLiD base plate when the shutter blades are released.

\subsection{SuFI CCD Sensor and Camera}

The camera of SuFI must fulfill the following key requirements: it must be UV sensitive down to $210 \mathrm{~nm}$, and it must combine a large format of $2048 \times 2048$ pixels with a fast read-out speed, such that at least one full frame per second can be read. We have chosen a PixelVision BioXight BV20CCD camera, based on a SITe S100AB-04 CCD with $2048 \times$ 2048 pixels and $12 \mu \mathrm{m} \times 12 \mu \mathrm{m}$ pixel pitch. The sensor is backside illuminated. It is used in full frame mode, with four output ports. 
Figure 8 Nikon D2H

focal-plane shutter as used in the SuFI instrument.

Figure 9 Cock mechanism for the SuFI shutter.
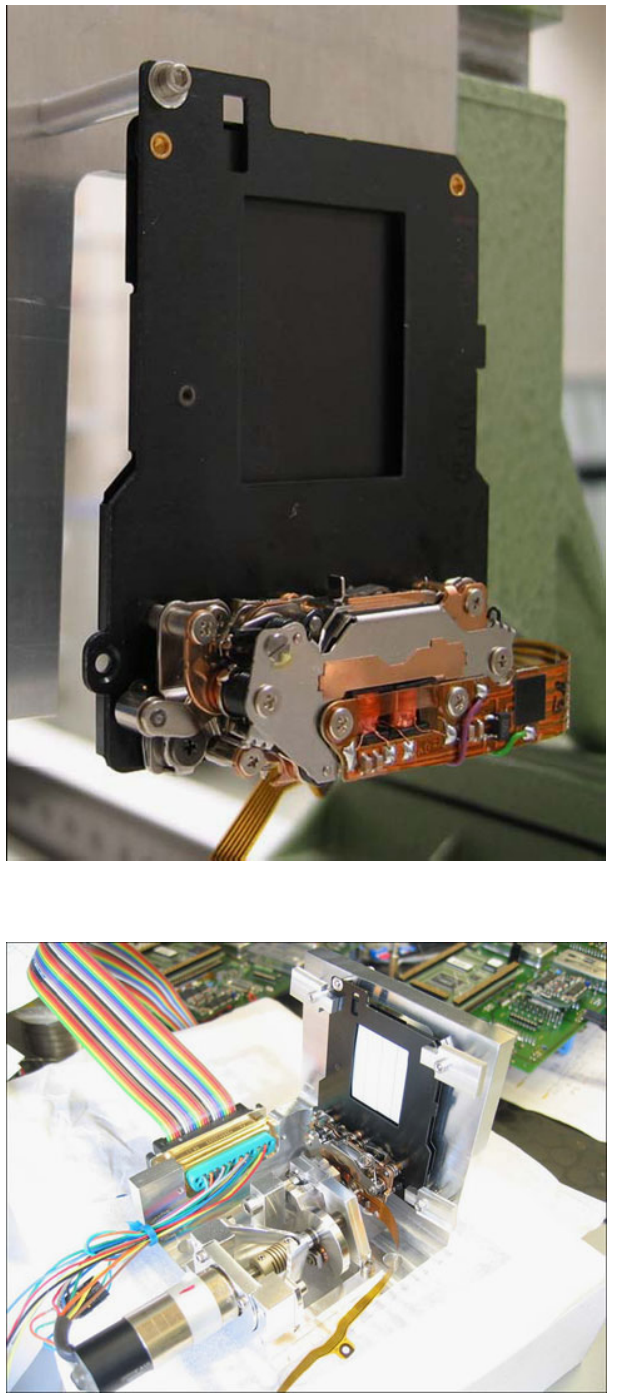

The camera electronics provide 14-bit digitization at a frame rate of a maximum of 2.64 frames per second. The commercial version of this camera employs a thermoelectric cooler with a Peltier element and with liquid cooling of the Peltier warm side. Specified dark current is 1.82 electrons per pixel per second at a CCD temperature of $240 \mathrm{~K}$.

For utilization in Sunrise the camera was heavily modified: The camera head (CCD) and camera electronics were separated in order to minimize heating of the camera head by power dissipation in the camera electronics. This separation allows two independent temperatures and cooling systems for the two subsystems.

While the camera head is cooled to about $270 \mathrm{~K}$ by two custom made methanol heat pipes (by Advanced Cooling Technologies (ACT), USA) directly connecting the warm side of the Peltier element with a distinct radiator, the camera electronics is housed in a pressure vessel, one side of which is used directly as a radiator. In this way the camera electronics 


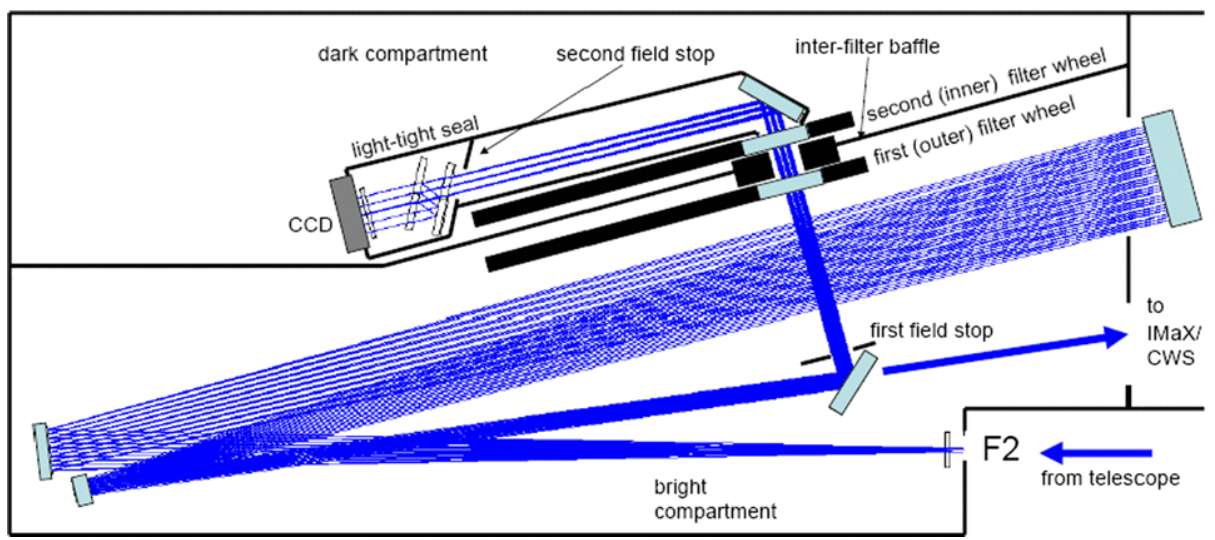

Figure 10 Scheme of the baffling system of SuFI. The light-tight innermost seal covers the light path after the second filtering, which is most vulnerable to parasitic illumination. The double filter wheel separates the dark compartment from the bright compartment. Two consecutive field stops narrow the field which is propagated to the detector.

(and thus the radiator) can operate at a significantly higher temperature than the CCD head, resulting in a high cooling efficiency of the radiator.

Electric connection between CCD and camera electronics is established by vacuum feedthroughs.

\subsection{Straylight Control}

The solar surface represents an object with relatively low intrinsic intensity contrast. The observed contrast values are further diminished by optical aberrations (see e.g. Danilovic et $a l ., 2008$ ) and instrument stray light (see e.g. Mathew, Zakharov, and Solanki, 2009). Both factors are of increasing importance when observing in the UV for different reasons: optical aberrations due to optical surface imperfections scale with $\lambda^{-1}$. At the same time spatial scattering (angular redistribution at optical surfaces) is typically also more pronounced at shorter wavelengths. The most important source of straylight in solar UV imagers is, however, spectral contamination by photons of the longer wavelength parts of the photospheric spectrum, which can be orders of magnitude more intense than the photon contributions within the selected UV science passband. To suppress these contaminations two identical filters in tandem are used, which has the additional advantage of providing a more "rectangular" band pass shape. Behind the filters the beam is highly sensitive to parasitic light and must therefore be completely light-tight. This is ensured by a baffle tube between filter wheel and camera head. Folding mirror, shutter assembly, and phase-diversity image doubler are all contained in this tube system.

In addition to this innermost seal, the whole ISLiD unit is divided into several compartments, which are separated by baffles and walls. The baffling concept is depicted in Figure 10. The first compartment is the brightest one. It serves as a light trap for the unused portions of the telescope field of view of $180 \mathrm{arcsec}$, which illuminates the tip-tilt mirror but is not completely picked up by the limited field of view of the Schwarzschild system (60 arcsec). This is still significantly larger than the SuFI science field of view of $17^{\prime \prime} \times 35^{\prime \prime}$. Therefore, another mask is placed in the reflected beam of the UV separator, such that the filter wheel is illuminated by a slightly vignetted field of $20^{\prime \prime} \times 40^{\prime \prime}$ only, with $17^{\prime \prime} \times 35^{\prime \prime}$ 
unvignetted. The filter wheel provides a separation between the bright compartment and a dark compartment, which is a closed box containing the inner filter wheel and the camera head, together with the above-mentioned light-tight innermost baffling tube. The first filter wheel resides in the bright compartment, and a baffle tube between the two filter wheels forms the optical feed-through from one compartment into the next.

\section{Structural and Mechanical Design of SuFI/ISLiD}

\subsection{Post-focus Instrumentation Platform Concept}

ISLiD/SuFI, CWS, and IMaX are built as individual optomechanical subunits (modules). The general design approach of the Post-focus Instrumentation platform (PFI) is to accommodate, implement, and align these individual modules into the PFI support structure. All modules are internally aligned and tested before integration into the PFI support structure. The concept of individual modules has the advantage that each module can be designed to achieve maximum internal stiffness, which helps to minimize internal alignment errors due to changing gravity load (elevation change). The IMaX and CWS modules are isostatically mounted into the support structure. Forces arising from differential thermal expansion between the modules and the PFI thus do not lead to mechanical tensions in the modules. Relative lateral alignment of the individual modules within the support structure must be guaranteed within limits of $\pm 0.2 \mathrm{~mm}$ in all directions. This requirement arises from the maximum image shift that can be tolerated at the optical interfaces between the modules. Angular alignment of the modules with respect to the fixation point (boresight error) must be ensured within a limit of 3 arcmin in all directions, compliant with a maximum relative pupil shift of $5 \%$ of the pupil diameter.

\subsubsection{ISLiD as Alignment Master Unit}

Since ISLiD has four optical interfaces, each described in position and in/out beam direction, it is convenient to use ISLiD as the 'alignment master': ISLiD is mounted rigidly within the instrument platform PFI; the relative alignment of ISLiD with respect to the telescope is done in the opposite sense: the telescope is aligned to ISLiD/PFI via the plane folding mirrors M3 and M4, which help in adjusting the incoming beam position (lateral as well as focus) and beam direction (pupil position). The ISLiD entrance F2 position is exclusively determined via the internal alignment of ISLiD (internal foci and stops) and may not be regarded as absolute in position. It is used as a focus compensator during alignment.

\subsubsection{PFI Mechanical Structure}

In order to maintain the optical alignment of the modules within the PFI for different thermal and mechanical load cases during flight, the PFI is built from carbon fiber reinforced plastic (CFRP) honeycomb plates, which are connected via aluminum inserts and screws. Such a structure has a very high stiffness-to-weight ratio.

\subsubsection{Thermal Aspects}

The CFRP plates were designed for minimum thermal expansion. The ISLiD optical bench plate (made from the same material) provides an additional stiffening of the PFI. The CWS 
base plate is also made from the same material, mitigating relative thermal expansion of the module within the PFI. Only the IMaX module is made from aluminum honeycomb; however, the effects of relative thermal expansion is uncritical, owing to the isostatic mounting of the IMaX module within the PFI platform. The Schwarzschild system itself is not perfectly athermal. It is based on Schott DURAN glass for the Schwarzschild mirrors in combination with the low thermoelastic expansion coefficient of the CFRP baseplate. The refractive reimaging paths feeding IMaX and CWS, respectively, make use of aluminum spacers to compensate the change in refractive index of the glass used for the lenses. The most important aspect is, however, that the two feed paths of IMaX and CWS are identically constructed and put to close vicinity, which minimizes the risk of a relative defocus between the two modules even in case of temperature inhomogeneities within the PFI.

The cameras and electronics inside the PFI need to be radiatively cooled. The PFI modules are therefore equipped with radiators tailored to achieve acceptable operating temperatures for the camera head (between $-5^{\circ} \mathrm{C}$ and $+10^{\circ} \mathrm{C}$ ) as well as for the electronic components (below $45^{\circ} \mathrm{C}$ ) nearby. The required radiator sizes and surface properties were derived from thermal analysis taking into account the flight scenario and the required operating temperatures.

\section{Instrument Operation, Observing Modes, and Science Data Products}

\subsection{Observing Modes}

The SuFI software allows for flexible observing programs. Observing modes differ basically in the wavelengths which are observed. For example, the shortest wavelength of $214 \mathrm{~nm}$ should only be observed when the elevation of the Sun is larger than 30 degrees above the horizon, and when the balloon altitude is above $36 \mathrm{~km}$ in order to minimize atmospheric extinction. By skipping the shortest wavelength the effective cadence of the other wavelength observations can be increased from one exposure per $40 \mathrm{~s}$ to one exposure per $8 \mathrm{~s}$ for a full wavelength cycle. For highest cadence observations a fixed wavelength observing mode is available for each wavelength (one exposure per second). Also an alternating wavelength mode between two or more filter positions is possible for each combination of wavelengths. Besides the used wavelengths with their specific exposure times, there are several other factors that limit the image cadence: the filter changing time (1 s), the cocking time of the mechanical shutter $(0.5 \mathrm{~s})$, the CCD read-out time $(0.4 \mathrm{~s})$, the execution time of the image compression, the network bandwidth, and the performance of the SuFI and the Instrument Control Unit (ICU) software.

\subsection{Software Design}

The system design of SuFI is mainly driven by the requirements coming from the PixelVision camera, which, for example, constrains the operating system to Microsoft Windows 2000. Other subsystems of the Sunrise mission are constrained to other operating systems, which leads to a heterogeneous software environment. In a nearly autonomous mission of six days duration, robustness and reliability of all system components, in particular of all software components, are mandatory. We therefore use the Adaptive Communication Environment (ACE) library, whose reliability is well proven by many applications running on several platforms over more than a decade (Schmidt and Huston, 2002, 2003). The core of the pattern-oriented ACE library is a thread pool reactor (Schmidt et al., 2000) that transfers scientific images as well as house-keeping data over the $100 \mathrm{MBit} \mathrm{s}^{-1}$ Ethernet network 
connection to the ICU, which processes the data and stores them on one of two data storage subsystems (DSS) via an IEEE 1394 connection. Optionally, data are sent to the ground over one of two telemetry channels. Additionally, the reactor receives commands from two possible command sources:

i) Commands can be sent from a timeline-driven observing program stored on the ICU before launch.

ii) Commands can be sent manually over a telemetry channel from the ground.

Since multithreaded programming in a distributed system is difficult and error-prone, we developed a memory checking tool to detect memory leaks automatically before launch as well as a call stack logger tool to detect deadlocks. Our aim of a maximum performance of the SuFI software (i.e. the maximum image cadence) is reached by a profiling tool that measures the execution time of each critical part of the software. Our development of both, the call stack logger and the profiler, is based on the technique of method call interception (Sayfan, 2005).

\subsection{SuFI Electronics Unit}

The SuFI Electronics Unit (SuFI-EU) is an IBM compatible computer system with all the interfaces needed to connect the other hardware components of the SuFI instrument. The heart of the SuFI-EU box is an AMPRO LittleBoard 800 mainboard, featuring an Intel $1.4 \mathrm{GHz}$ LV Pentium M 738, 1 GB RAM, 4 GB flash disk boot device. A 100 MBit Ethernet port connects the ICU, a PCI slot connects the PixelVision Lion 2 frame grabber card, which provides the interface to the CCD camera. A serial port RS422 connects the SuFI mechanism controller responsible for the filter wheel, the shutter, and the camera's exposure, and a serial port RS232 connects the internal Health Monitoring System for temperature and pressure monitoring and for control of the air circulation inside the hermetically sealed SuFI-EU box via three fans.

\subsection{Science Data Products}

During the six-day duration mission in June 2009 the SuFI instrument acquired about 150000 images. Each image is compressed and a header with a current set of house-keeping data is added. These raw data are transferred to the ICU, which stores them in a RAID level-5 format on the DSS. After the successful landing, the DSS was recovered, the RAID format was decoded, and the raw image data format was converted into the level-0 format using the Flexible Image Transport System (FITS). The FITS header consists of two sections of entries. The first section is the common part of SuFI and IMaX and contains system information like acquisition time, reduction level, pointing position, solar ephemeris data, GPS position and flight altitude, etc. The second section contains the instrument specific part and is different for SuFI and IMaX.

The level-0 science data are further processed by correcting for dark current and flat field (acquired in flight by averaging 100 exposures during a 10 min random walk on the solar surface using the coarse pointing system) and by removing some instrumental effects, which provides the level-1 FITS data. Finally, the phase-diversity reconstruction leads to the level-2 FITS data. 


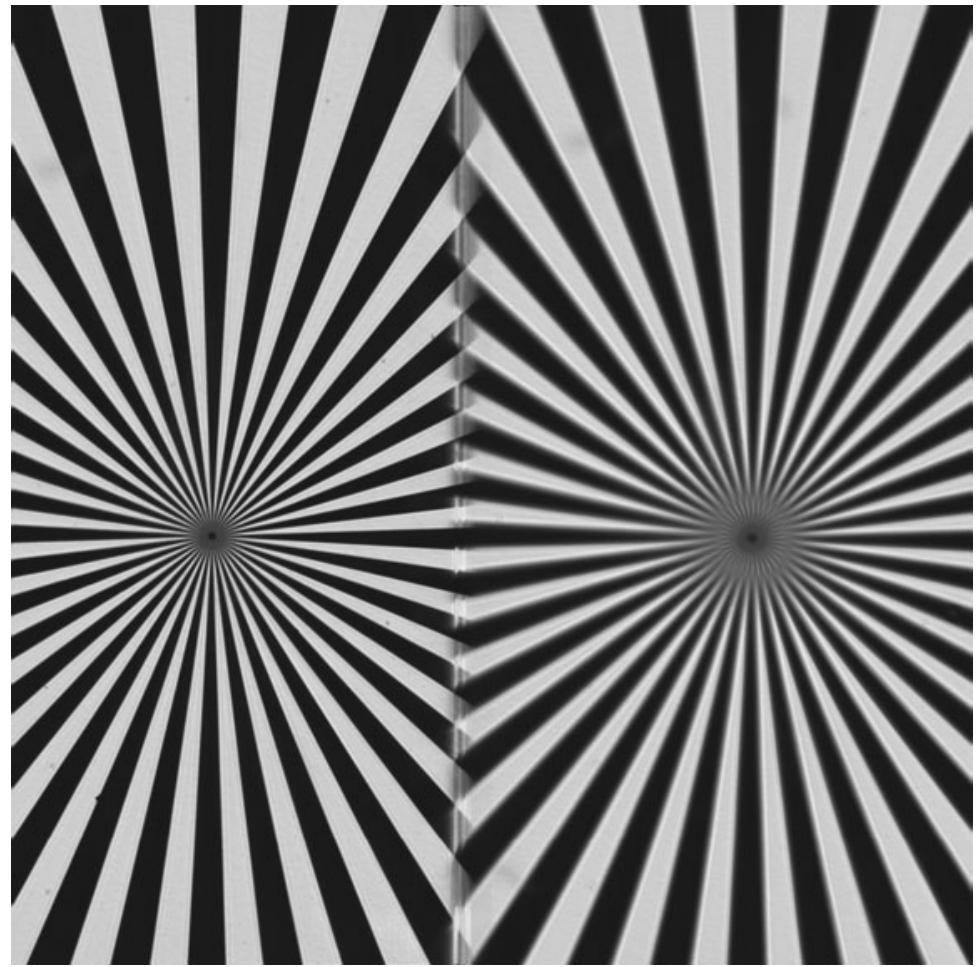

Figure 11 Image of a Siemens star target located in telescope F2 taken in the $388 \mathrm{~nm}$ channel. The differential defocus between the sharp image (left) and the defocussed image (right) is $\lambda / 2$. The cut-off frequency is consistent with the theoretical diffraction limit.

Figure 12 Detail from above, showing the innermost part of the in-focus image. The white circle indicates the classical Airy disk as defined as $1.22 \lambda / D$, which for the test wavelength of $388 \mathrm{~nm}$ corresponds to a radius of $0.1^{\prime \prime}$. At this wavelength the diffraction limit is strongly oversampled, which can clearly be seen.

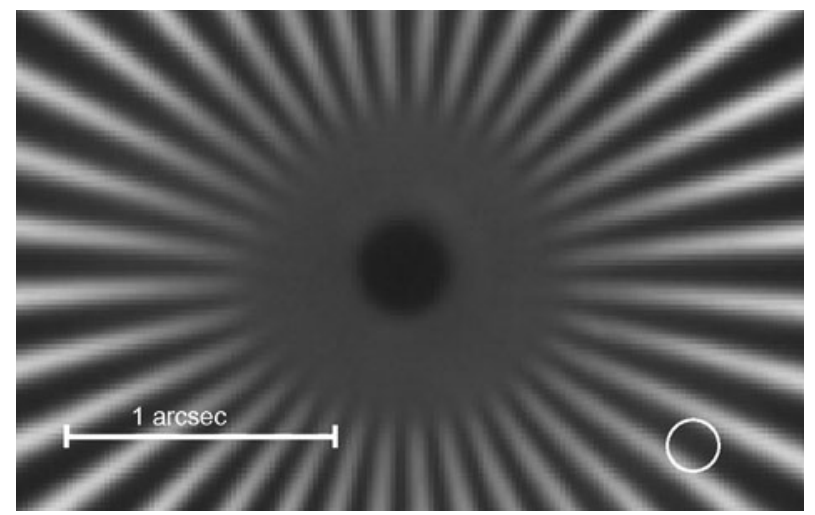

\section{Optical On-Ground Performance}

ISLiD/SuFI was tested and calibrated on ground prior to the science flight. The optical performance of SuFI was verified by inserting optical targets in F2 and analyzing the images of the targets taken by the SuFI camera. For the ISLiD-IMaX and ISLiD-CWS feed paths a dedicated camera placed in F4 and F6, respectively, was used. Contrast transfer and spatial 
resolution (cut-off frequency) was tested with a Siemens star target. Distortion was measured with the help of a rectangular grid target. Internal magnification and anamorphotic distortion were measured by a micrometer scale in F2. The optical quality of the ISLiDIMaX feed path was measured in terms of wavefront distortion by use of an interferometer in autocollimation setup, thus in double pass. At the measurement wavelength of $633 \mathrm{~nm}$, the rms wavefront error in double pass including the autocollimation sphere and the interferometer was $\lambda / 22$. Polarimetric properties of the ISLiD-IMaX feed path were characterized in terms of Mueller matrix maps over the full $\mathrm{IMaX}$ field of view by inserting polarization optics (rotatable linear polarizer and quarter wave plate) in front of F2 and measuring the full Stokes vector image at F4 with the Zurich imaging polarimeter ZIMPOL II (Gandorfer et al., 2004) at a wavelength of $525 \mathrm{~nm}$ selected by an interference filter of $5 \mathrm{~nm}$ width.

Both the polarimetric calibration and the interferometric test of the wavefront distortion confirmed the predicted performance. As seen from IMaX, ISLiD is basically invisible in terms of wavefront degradation as well as degradation of the very high IMaX polarimetric efficiencies (Martínez Pillet et al., 2010). Some optical key performance values are listed in Table 2.

\section{Conclusions}

The instrumental concept and design of ISLiD/SuFI was finally proven during the first scientific balloon flight of the Sunrise mission from 8 to 13 June 2008.

During the whole period the instrument performance was excellent. IMaX and SuFI were coaligned and both in focus, which was actively controlled by CWS (by focusing the telescope). Relative defocus between the different exit foci of ISLiD was always below $\lambda / 20$, well in the range that can be corrected for during phase-diversity treatment of the data. IMaX wavefront performance was not affected by ISLiD, and the polarimetric efficiency of IMaX was always nominal, i.e. the Mueller matrix of ISLiD was stable (Martínez Pillet et al., 2010).

SuFI collected a total of 150000 images in all wavelength bands. Except for a negligible number of excursions the SuFI shutter worked perfectly; the filter wheel had no functional problem at all.

SuFI collected the first high resolution (order 0.1 arcsec) images of the solar photosphere in the important wavelength range between $200 \mathrm{~nm}$ and $350 \mathrm{~nm}$, which is of particular interest for solar irradiance modeling.

A sample set of images at the different wavelengths after preliminary phase-diversity reconstruction (Hirzberger et al., 2010a) is plotted in Figure 13. A careful analysis of the data (Hirzberger et al., 2010a; Riethmüller et al., 2010; Solanki et al., 2010) reveals that the observed intensity contrasts are remarkably high, showing the extraordinary optical performance of the total system of telescope and instrument, especially the virtual lack of straylight contamination.

In the shortest wavelength channel at $214 \mathrm{~nm}$, exposure times were one or two orders of magnitude larger than anticipated, due to strong residual ozone extinction even in maximum flight altitude of $37.5 \mathrm{~km}$. Therefore the ultimate spatial resolution on the solar surface could not be reached during our science flight. This is partly due to the evolution of the solar structures during the long exposure time; to a larger extent, however, residual image smear on the order of 0.04 arcsec (rms) at frequencies in the tens of Hertz range corrupted the images also in the other wavelength bands, which have typical exposure times of $100 \mathrm{~ms}$. For a detailed analysis of the performance of the CWS system we here refer to Berkefeld $e t$ 
Table 2 Key optical performance parameters of ISLiD/SuFI.

\begin{tabular}{|c|c|c|c|c|}
\hline Item & Wavelength & Design goal & Measured & Comment \\
\hline \multirow[t]{2}{*}{$\begin{array}{l}\text { Internal } \\
\text { magnification }\end{array}$} & $500 \mathrm{~nm}$ & 1.05 & 1.041 & $\begin{array}{l}\text { Measured with } \\
\text { micrometer scale } \\
\text { in F2 and in F6 }\end{array}$ \\
\hline & $525 \mathrm{~nm}$ & 1.05 & 1.034 & $\begin{array}{l}\text { Measured with } \\
\text { micrometer scale } \\
\text { in F2 and in F4 }\end{array}$ \\
\hline \multirow[t]{3}{*}{ Transmittance } & $214 \mathrm{~nm}$ & $\geq 0.5$ & 0.52 & $\begin{array}{l}\text { Excluding filters; } \\
\text { calculated from } \\
\text { transmission } \\
\text { measurements of } \\
\text { components/samples }\end{array}$ \\
\hline & $525 \mathrm{~nm}$ & $\geq 0.5$ & 0.56 & $\begin{array}{l}\text { Measured } \\
\text { between F2-F4 }\end{array}$ \\
\hline & $500 \mathrm{~nm}$ & $\geq 0.5$ & 0.56 & $\begin{array}{l}\text { Measured } \\
\text { between F2-F6 }\end{array}$ \\
\hline $\begin{array}{l}\text { Linear } \\
\text { polarization }\end{array}$ & $525 \mathrm{~nm}$ & $\leq 0.1$ & 0.04 & Measured \\
\hline Distortion & $\begin{array}{l}\text { All } \\
\text { wavelengths }\end{array}$ & $\leq 0.01$ & $\leq 0.005$ & $\begin{array}{l}\text { Measured with } \\
\text { grid target in F2 }\end{array}$ \\
\hline $\begin{array}{l}\text { WFE (RMS) } \\
\text { on ground }\end{array}$ & $633 \mathrm{~nm}$ & & $\lambda / 22$ & $\begin{array}{l}\text { Measured in } \\
\text { double path at F2-F4; } \\
\text { focus removed }\end{array}$ \\
\hline $\begin{array}{l}\text { WFE (RMS) } \\
\text { in flight }\end{array}$ & $300 \mathrm{~nm}$ & & $\lambda / 17$ & $\begin{array}{l}\text { Deduced from } \\
\text { PD analysis } \\
\text { telescope removed }\end{array}$ \\
\hline $\begin{array}{l}\text { WFE (RMS) } \\
\text { in flight }\end{array}$ & $300 \mathrm{~nm}$ & & $\lambda / 5-6$ & $\begin{array}{l}\text { Deduced from } \\
\text { PD analysis } \\
\text { telescope included }\end{array}$ \\
\hline $\begin{array}{l}\text { Relative } \\
\text { defocus } \\
\text { SuFI-F6 }\end{array}$ & $500 \mathrm{~nm}$ & $\leq \lambda / 10$ & $\lambda / 20$ & $\begin{array}{l}\text { In-flight } \\
\text { performance, } \\
\text { from PD }\end{array}$ \\
\hline $\begin{array}{l}\text { Relative } \\
\text { defocus } \\
\text { SuFI-F4 }\end{array}$ & $525 \mathrm{~nm}$ & $\leq \lambda / 10$ & $\lambda / 20$ & $\begin{array}{l}\text { In-flight } \\
\text { performance, } \\
\text { from PD }\end{array}$ \\
\hline
\end{tabular}

al. (2010). From the phase-diversity reconstruction of the wavefront in the instruments exit pupil we have no indication of considerable optical imperfections, which could explain the limited angular resolution in our images (Hirzberger et al., 2010b). At $300 \mathrm{~nm}$ the in-flight rms WFE including the telescope is typically between $\lambda / 6$ and $\lambda / 5$. Since the telescope has $\lambda / 12$ at $633 \mathrm{~nm}$, this implies an rms WFE of SuFI/ISliD of better than $\lambda / 17$. For a detailed analysis of the PD reconstruction and the WFE analysis we here refer to Hirzberger et al. (2010b). 


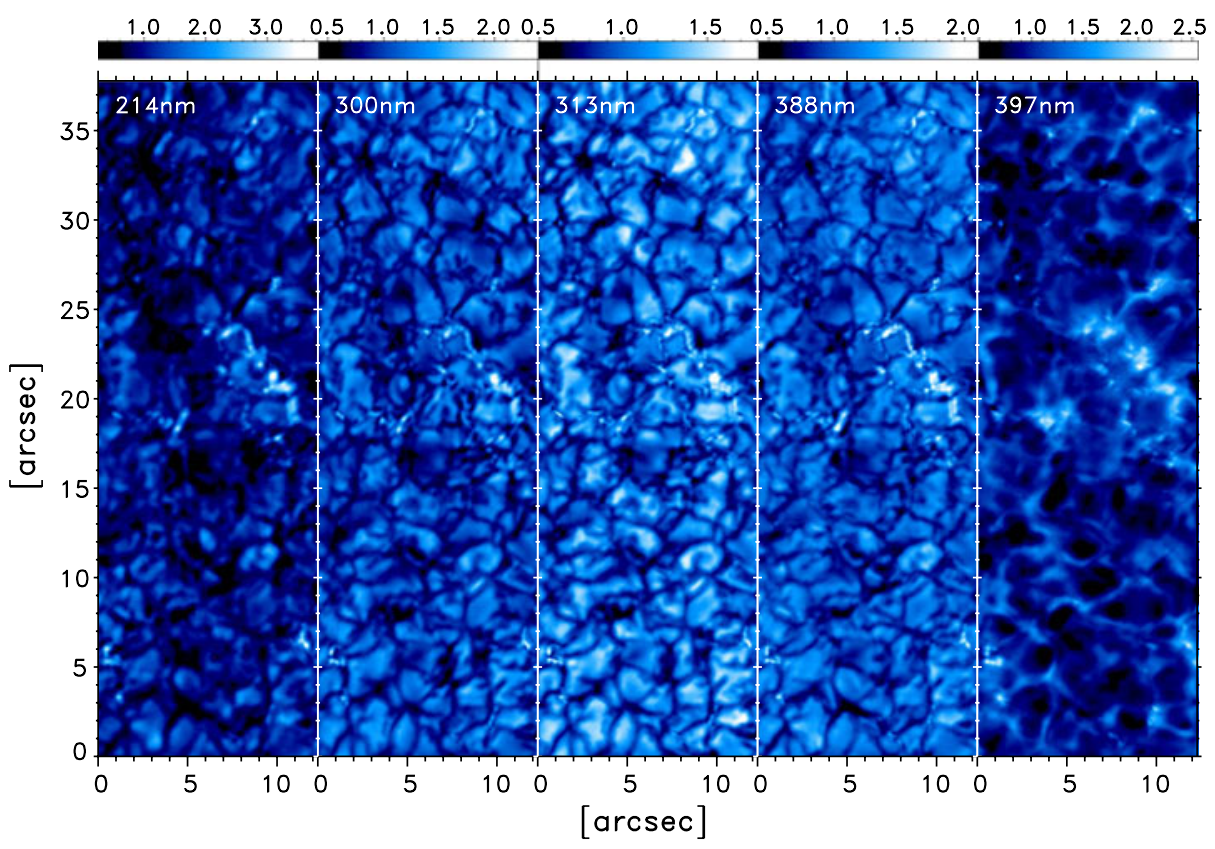

Figure 13 Phase-diversity reconstructed SuFI images of the solar surface. The field of view covers $1 / 20000$ of the total surface of the Sun. The depth of the false color table is always chosen to display the full intensity range. The remarkable contrasts in the UV wavelengths, which have been never observed before, represent an important ingredient for solar irradiance models.

Figure 14 Detail of a

phase-diversity reconstructed SuFI image of the solar surface at a wavelength of $300 \mathrm{~nm}$. The depth of the false color table is chosen to display the full intensity range.

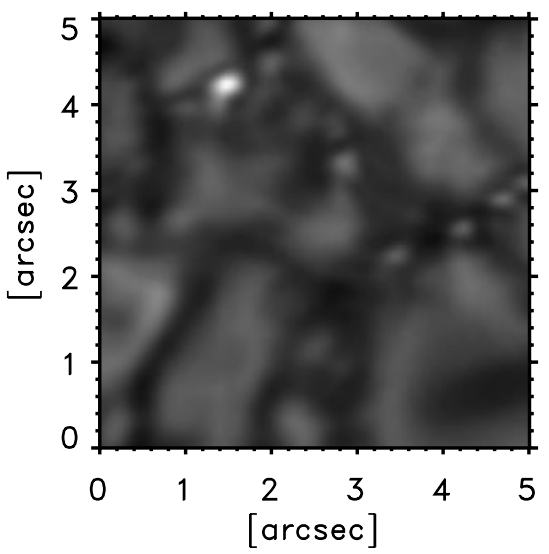

Although the CWS system could dramatically improve the effective pointing stability of the telescope, it was not able to completely damp the incoming disturbance spectrum of the balloon/gondola system. The effective angular resolution of SuFI is therefore between 0.1 arcsec and 0.15 arcsec. Although this is slightly above the theoretical diffraction limit, the quality of the SuFI data is resounding and the data set is expected to provide new insights in the physics of solar surface magnetism at very small scales. ISLiD/SuFI survived the flight and the landing with only negligible damage and are ready to be used again in Sunrise. 
Acknowledgements The German contribution to Sunrise is funded by the Bundesministerium für Wirtschaft und Technologie through Deutsches Zentrum für Luft- und Raumfahrt e.V. (DLR), Grant No. 50 OU 0401, and by the Innovationsfond of the President of the Max Planck Society (MPG). The Spanish contribution has been funded by the Spanish MICINN under projects ESP2006-13030-C06 and AYA200914105-C06 (including European FEDER funds). HAO/NCAR is sponsored by the National Science Foundation, and the HAO Contribution to Sunrise was partly funded through NASA grant number NNX08AH38G. We would like to thank the anonymous reviewer and the editor for very constructive and helpful comments on the manuscript.

Open Access This article is distributed under the terms of the Creative Commons Attribution Noncommercial License which permits any noncommercial use, distribution, and reproduction in any medium, provided the original author(s) and source are credited.

\section{References}

Barthol, P., Gandorfer, A., Solanki, S.K., Schüssler, M., Chares, B., Curdt, W., et al.: 2010, Solar Phys. this issue. doi:10.1007/s11207-010-9662-9

Berkefeld, T., Schmidt, W., Soltau, D., Bell, A., Doerr, H., Feger, B., et al.: 2010, Solar Phys. this issue.

Danilovic, S., Gandorfer, A., Lagg, A., Schüssler, M., Solanki, S.K., Vögler, A., Katsukawa, Y., Tsuneta, S.: 2008, Astron. Astrophys. 484, L17.

Löfdahl, M.G., Scharmer, G.B.: 1994, Astron. Astrophys. Suppl. 107, 243.

Gandorfer, A., Povel, H.P., Steiner, P., Aebersold, F., Egger, U., Feller, A., et al.: 2004, Astron. Astrophys. 422, 703.

Hirzberger, J., Feller, A., Riethmüller, T.L., Schüssler, M., Borrero, J.M., Afram, N., et al.: 2010a, Astrophys. J. 723, L154. doi:10.1088/2041-8205/723/2/L154

Hirzberger, J., Feller, A., Riethmüller, T.L., Gandorfer, A., Solanki, S.K.: 2010b, Astron. Astrophys. submitted.

Martínez Pillet, V., del Toro Iniesta, J.C., Álvarez-Herrero, A., Domingo, V., Bonet, J.A., González Fernández, L., et al.: 2010, Solar Phys. this issue. doi:10.1007/s11207-010-9644-y

Mathew, S.K., Zakharov, V., Solanki, S.K.: 2009, Astron. Astrophys. 501, L19.

Paxman, R.G., Schulz, T.J., Fienup, J.R.: 1992, J. Opt. Soc. Am. 9, 1072.

Riethmüller, T.L., Solanki, S.K., Martínez Pillet, V., Hirzberger, J., Feller, A., Bonet, J.A., et al.: 2010, Astrophys. J. 723, L169. doi:10.1088/2041-8205/723/2/L169

Sayfan, G.: 2005, C/C++ Users J. 23(4), 32.

Schmidt, D.C., Stal, M., Rohnert, H., Buschmann, F.: 2000, In: Pattern-Oriented Software Architecture: Patterns for Concurrent and Network Objects 2, Wiley, New York, 1.

Schmidt, D.C., Huston, S.D.: 2002, In: C++ Network Programming: Mastering Complexity with ACE and Patterns 1, Addison-Wesley, Boston, 21.

Schmidt, D.C., Huston, S.D.: 2003, In: C++ Network Programming: Systematic Reuse with ACE and Frameworks 2, Addison-Wesley, Boston, 39.

Solanki, S.K., Barthol, P., Danilovic, S., Feller, A., Gandorfer, A., Hirzberger, J., et al.: 2010, Astrophys. J. 723, L127. doi:10.1088/2041-8205/723/2/L127 